\title{
Scalable Aerobic Oxidation of Alcohols Using Catalytic DDQ/HNO
}

\author{
Tania Katsina ${ }^{\dagger}$ Louis Clavier, $^{\dagger}$ Jean-François Giffard ${ }^{\dagger}$ Nayane Macedo Portela da Silva, ${ }^{\star}$ \\ Jean Fournier, ${ }^{\ddagger}$ Rodolphe Tamion, ${ }^{\ddagger}$ Chloé Copin ${ }^{\star}$ Stellios Arseniyadis, ${ }^{*}{ }^{\dagger}$ and Alexandre Jean, ${ }^{*}$, \\ ${ }^{\dagger}$ Queen Mary University of London, School of Biological and Chemical Sciences. Mile End Road, E1 4NS, London, UK \\ * Industrial Research Centre, Oril Industrie, 13 rue Desgenétais, 76210, Bolbec, France.
}

Supporting Information

\begin{abstract}
A selective, practical and scalable aerobic oxidation of alcohols is described using catalytic amounts of 2,3-dichloro-5,6-dicyano-1,4-benzoquinone (DDQ) and $\mathrm{HNO}_{3}$, with molecular oxygen serving as the terminal oxidant. The method was successfully applied to the oxidation of a wide range of benzylic, propargylic and allylic alcohols, including two natural products, namely carveol and podophyllotoxin. The conditions are also applicable to the selective oxidative deprotection of PMB ethers.
\end{abstract}

KEYWORDS: Oxidation, alcohols, catalytic, DDQ, $\mathrm{HNO}_{3}$, aldehydes, ketones.

\section{INTRODUCTION}

Oxidation reactions play an important role in organic chemistry as showcased by the number of methods and reagents that have been developed over the years. ${ }^{1}$ More specifically, the selective oxidation of alcohols to the corresponding carbonyl compounds is a key transformation with broad applications in the pharmaceutical and agrochemical industry. ${ }^{2}$ Numerous protocols have been developed to perform such oxidations using either high oxidation state metal salts ${ }^{3}$ or various non-metallic oxidants such as hypervalent iodine, ${ }^{4} \mathrm{TEMPO}^{2 \mathrm{a}}$ and peroxides, ${ }^{2 \mathrm{~b}-\mathrm{c}}$ however these methods can present various drawbacks such as their cost or the inherent toxicity of the reagents used or the waste produced.

2.3-Dichloro-5,6-dicyano-1,4-benzoquinone (DDQ) $)^{5}$ is a well-known oxidant which has been successfully applied to numerous organic transformations, including alcohol oxidation, ${ }^{6}$ protecting group removal, ${ }^{7}$ aromatization, ${ }^{8}$ benzylic acetoxylation, ${ }^{9}$ oxidative coupling, ${ }^{10}$ oxidative cyclization $^{11}$ and biaryl construction ${ }^{12}$ just to name a few. Nonetheless, despite its efficiency, the stoichiometric use of DDQ often causes purification difficulties in large-scale syntheses, usually associated with the concomitant formation of the corresponding hydroquinone (DDHQ) in equimolar quantities. In addition, DDQ is relatively expensive and poses some
Figure 1. Oxidation methods using catalytic DDQ.
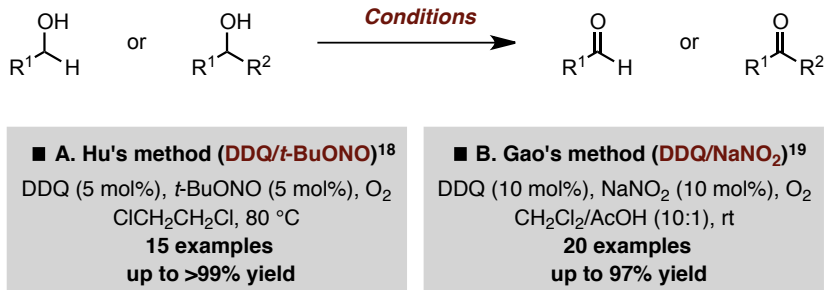

a. Hu's method (DDQ/t-BuONO) ${ }^{18}$ DDQ (5 mol\%), $t$-BuONO (5 mol\%), $\mathrm{O}_{2}$ $\mathrm{ClCH}_{2} \mathrm{CH}_{2} \mathrm{Cl}, 80^{\circ} \mathrm{C}$ 15 examples up to $>99 \%$ yield

- C. Moody's method (DDQ/NaNO ${ }_{2}$ or $\left.t-B u O N O / h v\right)^{20}$ DDQ (5 mol\%), $\mathrm{NaNO}_{2}$ or $t$-BuONO ( $\left.5 \mathrm{~mol} \%\right), \mathrm{O}_{2}$ $\mathrm{AcOH} / \mathrm{Dimethyl} \mathrm{carbonate,} \mathrm{rt}$, hv (visible light) 14 examples up to $>99 \%$ yield

D. Aerobic Oxidation of Alcohols Using Catalytic $\mathrm{DDQ} / \mathrm{HNO}_{3}$ (this work) $\mathrm{DDQ}(10$ or $20 \mathrm{~mol} \%), \mathrm{HNO}_{3}$ (20 or $\left.40 \mathrm{~mol} \%\right), \mathrm{O}_{2}$ DCM, it 46 examples up to $>99 \%$ yield

toxicity concerns due to the release of stoichiometric amounts of HCN upon workup. ${ }^{13}$

To address these limitations, a promising alternative is to combine DDQ with a less expensive, readily available and environmentally benign co-oxidant. In this context, several stoichiometric inorganic co-oxidants have been used in combination with catalytic amounts of DDQ, such as $\mathrm{MnO}_{2},{ }^{14} \mathrm{PbO}_{2},{ }^{14} \mathrm{Mn}(\mathrm{OAc})_{3},{ }^{15} \mathrm{Fe}\left(\mathrm{NO}_{3}\right)_{3}{ }^{16}$ and $\mathrm{FeCl}_{3} \cdot{ }^{7 \mathrm{a}, 17}$ The use of molecular oxygen as a readily available, non-toxic, inexpensive and more sustainable co-oxidant is an appealing alternative, unfortunately in the absence of any other redox compound, $\mathrm{O}_{2}$ fails to regenerate DDQ from DDHQ. Over the past years, various groups have been interested in developing an aerobic oxidation involving DDQ. Hu and co-workers for instance showed that tert-butyl nitrite (TBN) in conjunction with molecular oxygen could readily convert DDHQ into DDQ, and thus used this system to oxidize non-sterically hindered benzylic alcohols (Figure 1, A). ${ }^{18}$ In 2012, Gao and co-workers developed a practical process for the oxidation of alcohols to the corresponding carbonyl compounds using catalytic amounts of DDQ and $\mathrm{NaNO}_{2}$ and molecular oxygen as 
Table 1. Systematic study. ${ }^{a}$

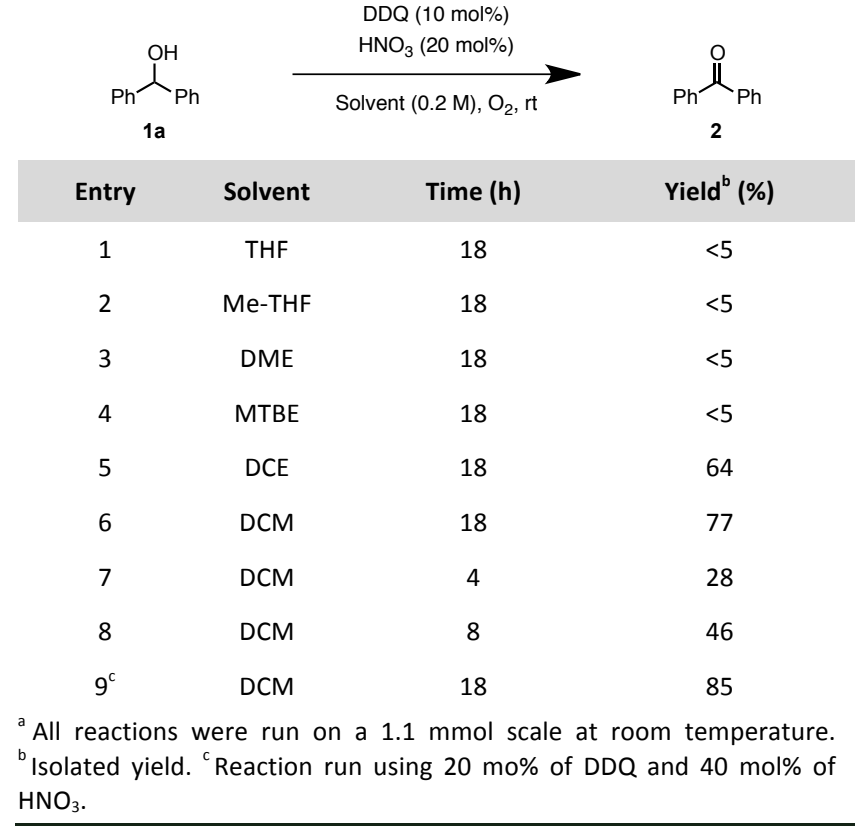

the terminal oxidant (Figure 1, B). ${ }^{19}$ Finally, Moody and co-workers developed a photochemical oxidation of benzylic and allylic alcohols using DDQ and sodium nitrite or tert-butyl nitrite (TBN) as co-catalysts and molecular oxygen as the terminal oxidant under visible light irradiation (Figure 1, C). ${ }^{20}$

Following the recent developments in the area, we became interested in developing a practical, cost-effective and scalable oxidation process using $\mathrm{HNO}_{3}$ as a source of $\mathrm{NO}$ to regenerate DDQ from DDHQ, and molecular oxygen as the terminal oxidant (Figure 1, D). We report here the results of our endeavour.

\section{RESULTS AND DISCUSSION}

We initiated this study using diphenyl methanol 1a as a model substrate running the reactions in a sealed tube using $10 \mathrm{~mol} \%$ of DDQ and $20 \mathrm{~mol} \%$ of $\mathrm{HNO}_{3}$ at room temperature for $18 \mathrm{~h}$. The results are summarized in Table 1.

A rapid evaluation of the set up showed that running the reaction in a sealed tube fitted with an oxygen balloon led to better results than running the reaction in an open flask or under molecular oxygen bubbling. The evaluation of a series of solvents also showed that THF, Me-THF, DME or even MTBE, which is ideal for industrial use, led to poor yields (Table 1, entries 1-4), while chlorinated solvent such as DCE and the DCM afforded the desired ketone 2 in 64 and $77 \%$ yield, respectively (Table 1, entries 5 and 6). The high yield and, most importantly, the low flammability of DCM compared to DCE, made this solvent particularly suitable for the development of a potential aerobic oxidation process.
Increasing the catalyst loading from $10 \mathrm{~mol} \%$ to $20 \mathrm{~mol} \%$ of DDQ and from $20 \mathrm{~mol} \%$ to $40 \mathrm{~mol} \%$ of $\mathrm{HNO}_{3}$ led to the best results with up to $85 \%$ isolated yield (Table 1, entry 9).

With these optimized conditions in hand [DDQ (20 mol\%), $\mathrm{HNO}_{3}$ (40 mol\%), DCM, rt, $18 \mathrm{~h}$ ], we next evaluated the substrate scope. It is worth pointing out however that some substrates were readily oxidized using only $10 \mathrm{~mol} \%$ of DDQ and $20 \mathrm{~mol} \%$ of $\mathrm{HNO}_{3}$. The results are summarized in Scheme 1.

A series of diarylmethanol were first subjected to the $\mathrm{DDQ} / \mathrm{HNO}_{3}$-catalyzed oxidation conditions. The corresponding diaryl ketones 2-16 were obtained in moderate to excellent yields mostly depending on the substitution pattern on the aromatic rings. As a general trend, the more electron-rich precursors led to the highest yields, with the para-methoxy derivatives $\mathbf{7}$ and $\mathbf{1 4}$ obtained in quasi-quantitative yield. More sterically hindered substrates such as the ones bearing a substituent at the ortho or the meta position led to lower yields compared to the corresponding para-substituted ones. Two arylheteroaryl alcohols were also evaluated. The reactivity of these heterocyclic substrates appeared to decrease when the basicity of the heterocycle increased as showcased by the higher yield obtained in the case of the thiophene derivative $(\mathbf{1 8}, 79 \%)$ compared to the furan $(17,40 \%)$.

A number of aryl alkyl methanol derivatives were also evaluated resulting in a similar trend, with the more electron-rich substrates such as para-methoxyethanol (22, 93\%), 1-(2H-1,3-benzodioxol-5-yl)ethan-1-ol (23, 77\%), 1-(4-biphenylyl)-1-ethanol (24, 90\%) and $\alpha$-methyl-2-naphthalenemethanol (26, 77\%) converted in good to excellent yields. Interestingly, the cyclopropyl derivative 21 was obtained without any noticeable ring opening albeit in a moderate $28 \%$ yield.

Various bicyclic aryl alkyl derivatives such as flavan-4-ol (28, 25\%), 1,2,3,4-tetrahydro-1-naphthol $(\mathbf{2 9}, 58 \%)$ and 1 -indanol $(\mathbf{3 0}, 55 \%)$ were also tested, however, these substrates appeared to be slightly less reactive as showcased by the lower conversions observed after $18 \mathrm{~h}$.

In an effort to generalize the method, aryl propargyl and aryl allyl alcohols were also tested and the corresponding ketones 31-35 were obtained in high yields ranging from $75 \%$ to $>99 \%$.

The method was also successfully applied to the synthesis of aromatic (36-40) and $\alpha, \beta$-unsaturated aldehydes (41-43), which were generally obtained in moderate to excellent yields starting from the corresponding benzylic and allylic alcohols.

The chemoselectivity of the reaction was also evaluated by applying our conditions to diol 44. As expected, only the benzylic alcohol was oxidized albeit in a low $15 \%$ yield. 


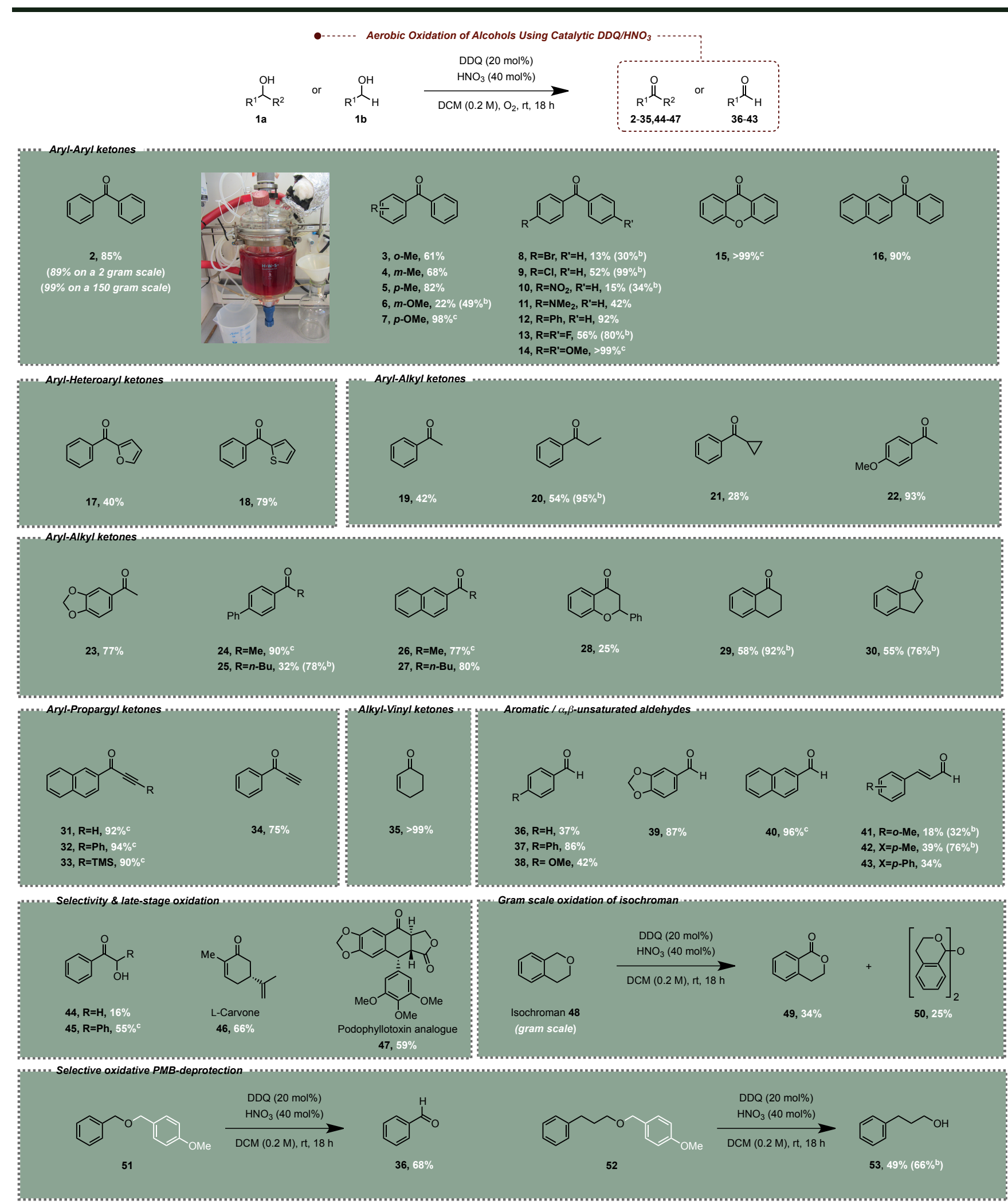

Scheme 1. ${ }^{a}$ All the reactions were run on a $0.5 \mathrm{mmol}$ scale unless otherwise noted using $20 \mathrm{~mol} \%$ of $\mathrm{DDQ}, 40 \mathrm{~mol} \%$ of $\mathrm{HNO}_{3}$, $\mathrm{O}_{2}$ (balloon), DCM, rt for $18 \mathrm{~h}$. ${ }^{\mathrm{b}}$ Yield based on recovered starting material (brsm). ${ }^{\mathrm{C}}$ The reactions were run using $10 \mathrm{~mol} \%$ of DDQ and $20 \mathrm{~mol} \%$ of $\mathrm{HNO}_{3}$.

With these results in hand and with the idea to showcase further the synthetic utility of the method, we applied our conditions to the oxidation of two natural products, namely L-carveol and podophyllotoxin. Both were converted to the corresponding ketones $\mathbf{4 6}$ and 47 in descent yields ranging from $59 \%$ to $66 \%$ 
(Scheme 1). The scope of the method could even be extended to isochroman derivatives, which also appeared to be good candidates. Indeed, subjecting isochroman 48 to our conditions led to isochromanone 49 in $34 \%$ yield, along with $25 \%$ of the corresponding dimer 50, which was quantitatively converted to ethoxyisochroman-2-one in the presence of ethanol.

Finally, following the work of $\mathrm{Hu}$ and co-workers on the oxidative deprotection of PMB ethers using their $\mathrm{DDQ} / t-\mathrm{BuONO} / \mathrm{O}_{2}$ system, ${ }^{18}$ we were wondering if our own system could reach the same outcome. To validate this hypothesis, we applied our conditions to PMBprotected benzyl alcohol 51. Interestingly, we were able to isolate benzaldehyde (36) in $68 \%$ yield, resulting from the deprotection and concomitant oxidation of benzyl alcohol. In contrast, when applying the conditions to ether $\mathbf{5 2}$, we only observed the formation of alcohol $\mathbf{5 3}$ in $49 \%$ yield, showcasing once again the chemoselectivity of our catalytic $\mathrm{DDQ} / \mathrm{HNO}_{3}$-mediated oxidation method.

After assessing the scope of the method, we eventually evaluated its scalability by performing a first reaction on a two-gram scale. To our delight, diphenyl methanol 1a was converted to the corresponding ketone 2 with a similar efficiency ( $89 \%$ yield). A much larger scale was also conducted (150 gram batch; $7 \mathrm{~L}$ reactor). The corresponding diphenyl ketone was obtained in $99 \%$ isolated yield after simple aqueous workup and concentration to dryness $(96.9 \%$ chemical purity determined by UPLC). It is worth pointing out that a solvent swap from DCM (reaction and extractive solvent) to any other solvent can easily be performed after the workup thus avoiding any isolation step. In order to minimise any potential risk associated with the use of oxygen, we used air instead.

Safety hazards have also been evaluated by DSC (done at the beginning of the reaction). The plot showed a weak exothermic phenomenon $\left(\Delta \mathrm{H}=-34 \mathrm{~J}_{\mathrm{g}} \mathrm{g}^{-1}\right)$ initiated at $184{ }^{\circ} \mathrm{C}\left(\mathrm{T}_{\text {onset }}\right)$ and extended until $267^{\circ} \mathrm{C}$, while the related enthalpy of decomposition showed a weak temperature rise of the mixture $\left(\Delta \mathrm{T}_{\mathrm{ad}}<50^{\circ} \mathrm{C}\right.$, adiabatic temperature rise) (see SI for more details). Evaluation of the thermal behavior of the reaction showed no rapid heat generation or rapid decomposition and no risk of explosive hazards could be detected. Overall, the process was ruled safe and amenable to scale-up.

The introduction of air by bubbling did however need to be assessed as it caused the release of hazardous nitrous gas outside the reactor which could potentially pose some health \& safety concerns but also some reactivity issues as reactive species are expelled from the reaction vessel during the process. To circumvent the latter issue, a second recharge of DDQ (0.1 equiv.) $/ \mathrm{HNO}_{3}$ ( 0.2 equiv.) was operated, which allowed to reach quasi complete conversion of the starting material ( $98 \%$ conv.). This gas releasing issue will be further examined for future developments. In
Figure 2. Proposed mechanism for the $\mathrm{DDQ} / \mathrm{HNO}_{3}$-mediated aerobic oxidation.

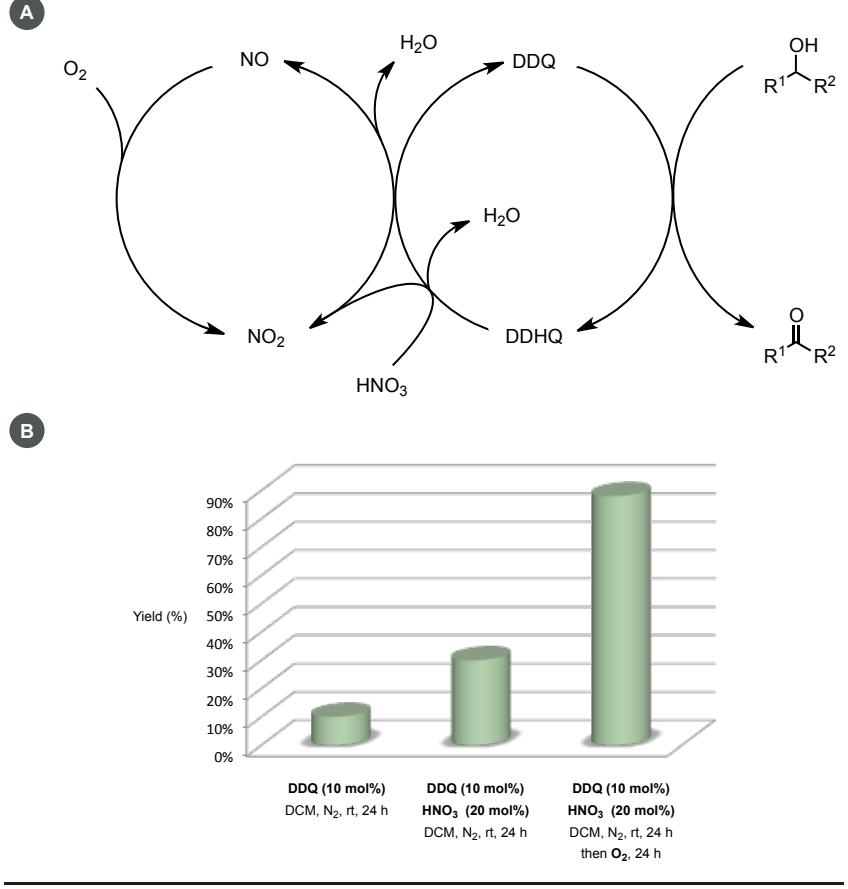

particular, we will look at implementing in pressure vessels and flow chemistry.

Based on the literature ${ }^{18,21}$ and on the results obtained so far, the following mechanism is proposed featuring two synergistic catalytic cycles where DDQ acts as a catalyst for the oxidation of the substrate, nitric acid as a source of $\mathrm{NO}_{2}$ that allows the regeneration of DDQ, and molecular oxygen which serves as a terminal oxidant. This mechanism is supported by the control experiments run under a nitrogen atmosphere using either $10 \mathrm{~mol} \%$ of DDQ or a combination of $10 \mathrm{~mol} \%$ of DDQ and $20 \mathrm{~mol} \%$ of $\mathrm{HNO}_{3}$ (Figure 2, B). It is worth pointing out that a full recovery of the reactivity is observed upon addition of molecular oxygen.

In conclusion, we have developed an efficient catalytic system using $\mathrm{DDQ} / \mathrm{HNO}_{3} / \mathrm{O}_{2}$ for the selective oxidation of benzylic, propargylic and allylic alcohols to the corresponding carbonyl compounds. The method is both practical and scalable and applicable to a wide range of alcohols. Interestingly, the same catalytic method can also be applied to the deprotection of PMB ethers.

\section{EXPERIMENTAL SECTION}

Typical procedure for the $\mathrm{DDQ} / \mathrm{HNO}_{3}$-catalysed oxidation. To a solution of the alcohol precursor (1 equiv.) in DCM $(0.2 \mathrm{M})$ at room temperature is added DDQ ( 0.2 equiv.) followed by a $2 \mathrm{M}$ aqueous solution of $\mathrm{HNO}_{3}$ (0.4 equiv.). The reaction vessel is then sealed and fitted with a balloon of $\mathrm{O}_{2}$. The reaction mixture is allowed to stir at the same temperature for $18 \mathrm{~h}$, time after which the reaction is quenched with a saturated aqueous solution of $\mathrm{NaHCO}_{3}$ and extracted with DCM. 
The combined organic phases are then dried over anhydrous $\mathrm{MgSO}_{4}$, filtered and evaporated under reduced pressure to afford a crude residue, which is purified by flash column chromatography over silica gel.

\section{ASSOCIATED CONTENT}

\section{Supporting Information}

The Supporting information is available free of charge on the Publications website. Details of experimental procedures, ${ }^{1} \mathrm{H}$ and ${ }^{13}$ C NMR spectra, DSC study.

\section{AUTHOR INFORMATION}

\section{Corresponding Author}

*E-mail: s.arseniyadis@qmul.ac.uk

*E-mail: alexandre.jean@servier.com

\section{ORCID}

Stellios Arseniyadis: 0000-0001-6831-2631

\section{Author Contributions}

The manuscript was written through contributions of all authors. All authors have given approval to the final version of the manuscript.

\section{Notes}

The authors declare no competing financial interest.

\section{ACKNOWLEDGMENT}

This research was supported by Queen Mary University of London and Oril Industrie.

\section{REFERENCES}

(1) (a) Bäckvall, J. E. Modern Oxidation Methods, VCH Wiley, Weinheim, 2004, pp 83-118. (b) Hundlucky, M. Oxidation in Organic Chemistry, American Chemical Society, Washington, DC, 1990. (c) Caron, S.; Dugger, R. W.; Ruggeri, S. G.; Ragan, J. A.; Ripin, D. H. B. Large-scale oxidations in the pharmaceutical industry. Chem. Rev. 2006, 106, 2943-2989.

(2) (a) Tojo, G.; Fernández, M. Oxidation of alcohols to aldehydes and ketones: A guide to current common practice; Springer Science Business Media: New York, 2010. (b) Haines, A. Methods for oxidation of organic compounds V2: Alcohols, alcohol derivatives, alkyl halides, nitroalkanes, alkyl azides, carbonyl compounds hydroxyarenes and aminoarenes; Academic Press: London, 1988. (c) Fuchs, P. L. Handbook of Reagents for Organic Synthesis: Catalytic Oxidation Reagents; Wiley \& Sons: Chichester, UK, 2013.

(3) Mallat, T.; Baiker, A. Oxidation of alcohols with molecular oxygen on solid catalysts. Chem. Rev. 2004, 104, 3037-3058.

(4) For reviews on hypervalent iodine reagents see: (a) Banks, D. F. Organic Polyvalent Iodine Compounds. Chem. Rev. 1966, 66, 243-266. (b) Stang, P. J.; Zhdankin, V. V. Organic polyvalent iodine compounds. Chem. Rev. 1996, 96, 1123-1178; (c) A. Varvoglis, Hypervalent iodine in organic synthesis; Academic Press: San Diego, 1997; (d) Stang, P. J.; Zhdankin, V. V. Recent developments in the chemistry of polyvalent iodine compounds. Chem. Rev. 2002, 102, 2523-2584; (e) Hypervalent Iodine Chemistry; Wirth, T., Ed.; Springer: Heidelberg, 2003.

(5) (a) Bharate, S. B. 2,3-Dichloro-5,6-dicyano-1,4-benzoquinone (DDQ). Synlett 2006, 496-497. (b) Walker, D.; Hiebert, J. D. 2,3-Dichloro-5,6-dicyanobenzoquinone and its reactions. Chem. Rev. 1967, 67, 153-195

(6) For selected examples of alcohol oxidation, see: (a) Peng, K.; Chen, F. X.; She, X. G.; Yang, C. H.; Cui, Y. X.; Pan, X. F. Selective oxidation of benzylic or allylic hydroxyl group of sec-1,2-diols. Tetrahedron Lett. 2005, 46, 1217-1220. (b) Mori, K.; Koseki, K. Synthesis of trichostatin a, a potent differentiation inducer of friend leukemic cells, and its antipode. Tetrahedron 1988, 44, 6013-6020. (c) McKittrick, B. A.; Ganem, B. Advances in the chemistry of 2,3epoxyalcohols. New methodology for contra-trans-diaxial opening and selective enediol oxidation. J. Org. Chem. 1985, 50, 5897-5898.

(7) For selected examples of protecting group removal, see: (a) Chandrasekhar, S.; Sumithra, G.; Yadav, J. S. Deprotection of mono and dimethoxy phenyl methyl ethers using catalytic amounts of DDQ. Tetrahedron Lett. 1996, 37, 1645-1646. (b) Rahim, M. A.; Matsumura, S.; Toshima, K. Photo-induced aromatic assembly of benzocycloalka[1,2- $b$ ] furan and spiro[furan-2(3H), 1'-benzocycloalkane] derivatives. Tetrahedron Lett. 2005, 46, 7307-7309. (c) Horita, K.; Yoshioka, T.; Tanaka, T.; Oikawa, Y.; Yonemitsu, O. On the selectivity of deprotection of benzyl, mpm (4-methoxybenzyl) and dmpm (3,4-dimethoxybenzyl) protecting groups for hydroxy functions. Tetrahedron, 1986, 42, 3021-3028.

(8) For selected examples of aromatization, see: (a) Ohmura, T.; Masuda, K.; Takase, I.; Suginome, M. Palladium-catalyzed silylene1,3-diene [4+1] cycloaddition with use of (aminosilyl)boronic esters as synthetic equivalents of silylene. J. Am. Chem. Soc. 2009, 131, 16624-16625. (b) Manning, J. R.; Davies, H. M. L. One-pot synthesis of highly functionalized pyridines via a rhodium carbenoid induced ring expansion of isoxazoles. J. Am. Chem. Soc. 2008, 130, 86028603. (c) Ploypradith, P.; Petchmanee, T.; Sahakitpichan, P.; Litvinas, N. D.; Ruchirawat, S. Total synthesis of natural and unnatural lamellarins with saturated and unsaturated D-rings. J. Org. Chem. 2006, 71, 9440-9448. (d) Shimizu, M.; Takahashi, A.; Kawai, S. Efficient pyrrole synthesis using double nucleophilic addition to $\alpha, \beta$-unsaturated imines with plural nucleophiles. Org. Lett. 2006, 8, 3585-3587. (e) Pla, D.; Marchal, A.; Olsen, C. A.; Albericio, F.; Alvarez, M. Modular total synthesis of lamellarin D. J. Org. Chem. 2005, 70, 8231-8234. (f) Wurz, R. P.; Charette, A. B. Doubly activated cyclopropanes as synthetic precursors for the preparation of 4-nitro- and 4-cyano-dihydropyrroles and pyrroles. Org. Lett. 2005, 7, 2313-2316.

(9) For selected examples of benzylic acetoxylation, see: (a) Kumar, V.; Sharma, A.; Sharma, M.; Sharma, U. K.; Sinha, A. K. DDQ catalyzed benzylic acetoxylation of arylalkanes: a case of exquisitely controlled oxidation under sonochemical activation. Tetrahedron 2007, 63, 9718-9723. (b) Marcantoni, E.; Petrini, M.; Profeta, R. Synthesis of advanced intermediates for the preparation of aza-analogues of podophyllotoxin. Tetrahedron Lett. 2004, 45, 2133 2136.

(10) Li, C. J. Cross-dehydrogenative coupling (CDC): Exploring $\mathrm{C}-\mathrm{C}$ bond formations beyond functional group transformations. Acc. Chem. Res. 2009, 42, 335-344.

(11) For selected examples of oxidative cyclizations, see: (a) Liu, L.; Floreancig, P. E. Cyclization reactions through DDQ-mediated vinyl oxazolidinone oxidation. Org. Lett. 2009, 11, 3152-3155. (b) Tu, W. Y.; Floreancig, P. E. Oxidative carbocation formation in macrocycles: Synthesis of the neopeltolide macrocycle. Angew. Chem. Int. Ed. 2009, 48, 4567-4571.

(12) Zhai, L.; Shukla, R.; Rathore, R. Oxidative $\mathrm{C}-\mathrm{C}$ bond formation (Scholl reaction) with DDQ as an efficient and easily recyclable oxidant. Org. Lett. 2009, 11, 3474-3477.

(13) $\mathrm{LD}_{50}$ of $82 \mathrm{mg} / \mathrm{kg}$.

(14) Liu, L.; Floreancig, P. E. 2,3-Dichloro-5,6-dicyano-1,4benzoquinone-catalyzed reactions employing $\mathrm{MnO}_{2}$ as a stoichiometric oxidant. Org. Lett. 2010, 12, 4686-4689.

(15) Cosner, C. C.; Cabreara, P. J.; Byrd, K. M.; Thomas, A. M. A.; Helquist, P. Selective oxidation of benzylic and allylic alcohols using $\mathrm{Mn}(\mathrm{OAc})_{3} /$ catalytic 2,3-dichloro-5,6-dicyano-1,4benzoquinone. Org. Lett. 2011, 13, 2071-2073.

(16) $\mathrm{Hu}, \mathrm{Y}$.; Chen, L.; Li, B. Fe( $\left(\mathrm{NO}_{3}\right)_{3} / 2,3$-dichloro-5,6-dicyano1,4-benzoquinone (DDQ): An efficient catalyst system for selective oxidation of alcohols under aerobic conditions. Catal. Commun. 2018 , $103,42-46$.

(17) Mozina, S.; Iskra, J. Aerobic oxidation of secondary alcohols with nitric acid and iron(III) chloride as catalysts in fluorinated alcohol. J. Org. Chem. 2019, 84, 14579-14586.

(18) Shen, Z.; Dai, J.; Xiong, J.; He, X.; Mo, W.; Hu, B.; Sun, N.; $\mathrm{Hu}, \mathrm{X}$. 2,3-Dichloro-5,6-dicyano-1,4-benzoquinone (DDQ)/tert-butyl 
nitrite/oxygen: A versatile catalytic oxidation system. Adv. Synth Catal. 2011, 353, 3031-3038.

(19) Wang, L.; Li, J.; Yang, H.; Lv, Y.; Gao, S. Selective oxidation of unsaturated alcohols catalyzed by sodium nitrite and 2,3-dichloro5,6-dicyano-1,4-benzoquinone with molecular oxygen under mild conditions. J. Org. Chem. 2012, 77, 790-794.

(20) Walsh, K.; Sneddon, H. F.; Moody, C. J. Solar photochemical oxidations of benzylic and allylic alcohols using catalytic organo- oxidation with DDQ: Application to lignin models. Org. Lett. 2014, $16,5224-5227$.

(21) Zhang, W.; Ma, H.; Zhou, L.; Sun, Z.; Du, Z.; Miao, H.; Xu, J. Organocatalytic oxidative dehydrogenation of dihydroarenes by dioxygen using 2,3-dichloro-5,6-dicyano-benzoquinone (DDQ) and $\mathrm{NaNO}_{2}$. Molecules 2008, 13, 3236-3245. 


\section{TABLE OF CONTENTS GRAPHIC}

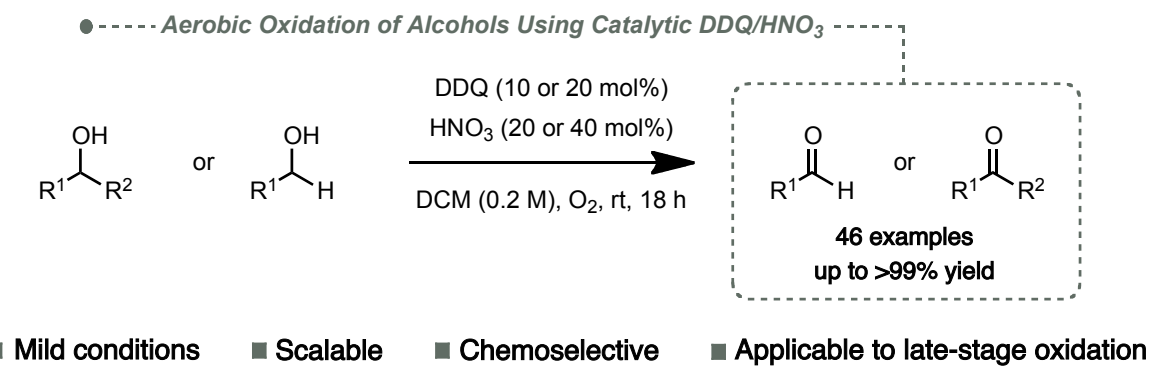

